\title{
The Peculiar Extinction of Herschel 36
}

\author{
Prepared by \\ J. H. HECHT \\ Space Sciences Laboratory \\ The Aerospace Corporation \\ H. L. HELFER, J. WOLF, and J. L. PIPHER \\ Department of Physics and Astronomy \\ University of Rochester \\ Rochester, New York 14627 \\ and \\ B. DONN \\ Laboratory for Extraterrestrial Physics \\ NASA/Goddard Space Flight Center \\ Greenbelt, Maryland 20771 \\ 1 October 1982 \\ VICE PRESIDENT AND GENERAL MANAGER \\ VICE PRESIDENT AND GENERAL MANAGER
Laboratory Operations

\footnotetext{
Prepared for
}

LIBRARY COPY

กCT 181982
LANGLEY RESEARCH CENTER LIBRA.RY, NASA

HAMPTON, VIRGINIA

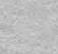




\section{LABORATORY OPERATIONS}

The Laboratory Operations of The Aerospace Corporation is conducting experimental and theoretical investigations necessary for the evaluation and application of scientific advances to new military space systems. Versatility and flexibility have been developed to a high degree by the laboratory personnel in dealing with the many problems encountered in the nation's rapidly developing space systems. Expertise in the latest scientific developments is vital to the accomplishment of tasks related to these problems. The laboratories that contribute to this research are:

Aerophysics Laboratory: Launch vehicle and reentry aerodynamics and heat transfer, propulsion chemistry and fluid mechanics, structural mechanics, flight dynamics; high-temperature thermomechanics, gas kinetics and radiation; research in environmental chemistry and contamination; $\mathrm{cw}$ and pulsed chemical laser development including chemical kinetics, spectroscopy, optical resonators and beam pointing, atmospheric propagation, laser effects and countermeasures.

Chemistry and Physics Laboratory: Atmospheric chemical reactions, atmospheric optics, light scattering, state-specific chemical reactions and radiation transport in rocket plumes, applied laser spectroscopy, laser chemistry, battery electrochemistry, space vacuum and radiation effects on materials, lubrication and surface phenomena, thermionic emission, photosensitive materials and detectors, atomic frequency standards, and bioenvironmental research and monitoring.

Electronics Research Laboratory: Microelectronics, GaAs low-noise and power devices, semiconductor lasers, electromagnetic and optical propagation phenomena, quantum electronics, laser communications, lidar, and electro-optics; communication sciences, applied electronics, semiconductor crystal and device physics, radiometric imaging; millimeter-wave and microwave technology.

Information Sciences Research office: Program verification, program translation, performance-sensitive system design, distributed architectures for spaceborne computers, fault-tolerant computer systems, artificial intelligence, and microelectronics applications.

Materials Sciences Laboratory: Development of new materials: metal matrix composites, polymers, and new forms of carbon; component fallure analysis and reliability; fracture mechanics and stress corrosion; evaluation of materials in space environment; materials performance in space transportation systems; analysis of systems vulnerability and survivability in enemy-induced environments.

Space Sciences Laboratory: Atmospheric and lonospheric physics, radiation from the atmosphere, density and composition of the upper atmosphere, aurorae and airglow; magnetospheric physics, cosmic rays, generation and propagation of plasma waves in the magnetosphere; solar physics, infrared astronomy; the effects of nuclear explosions, magnetic storms, and solar activity on the earth's atmosphere, ionosphere, and magnetosphere; the effects of optical, electromagnetic, and particulate radiations in space on space systems. 
1 RNATR-82(8454)-3

015 PLA $23 / 2$

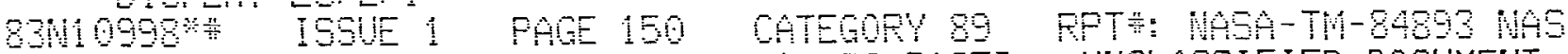

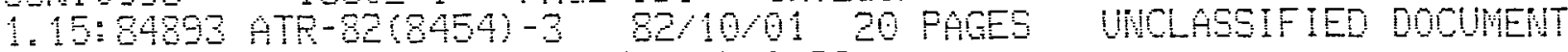

UT: The pedin extinction of Herschel 36

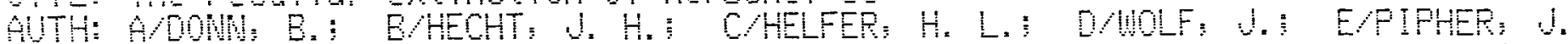

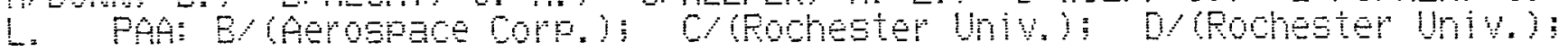
Elfogester Uhi.)

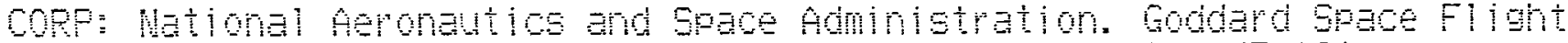

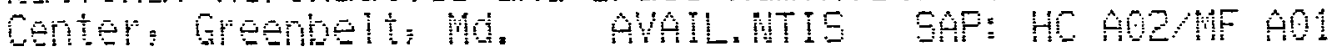

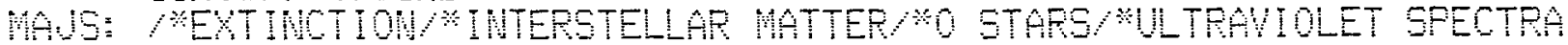

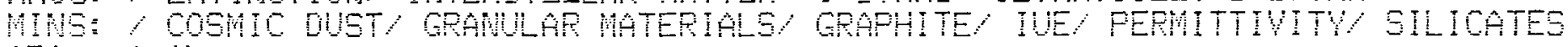

ABA: Author

ABs: The exthotion of Hershel 36 we mesured and toud to be peulin in the seme senes as thet oberved in orion. Following the treetment ot hath

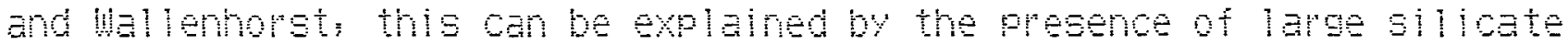

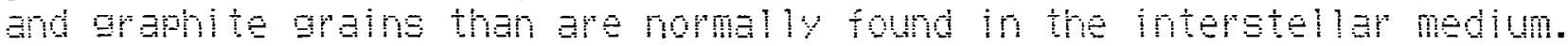
Corecting the stel ar fluk for foreground estinction results in a

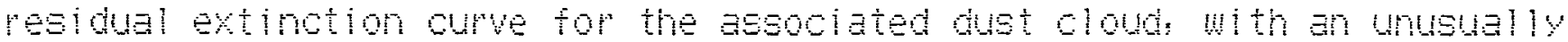
smel homel ized exthotion lese then 1.0$)$ at 1500 h. The lon Uy extinction mey be due to the efrects ot sotien by the dust cloud meter ial.

EMTEF: 
- - - 
Aerospace Report No. ATR-82(8454)-3

THE PECULIAR EXTINCTION OF HERSCHEL 36

Prepared by

J. H. Hecht

Space Sciences Laboratory

The Aerospace Corporation

H. L. Helfer, J. Wolf, and J. L. Pipher Department of Physics and Astronomy

University of Rochester

Rochester, New York 14627

and

B. Donn

Laboratory for Extraterrestrial Physics NASA/Goddard Space Flight Center

Greenbelt, Maryland 20771

1 October 1982

Laboratory Operations

THE AEROSPACE CORPORATION

E1 Segundo, California 90245

Prepared for

VICE PRESIDENT AND GENERAL MANAGER

Laboratory Operations 
$-$ 
THE PECULIAR EXTINCTION OF HERSCHEL 36

Prepared
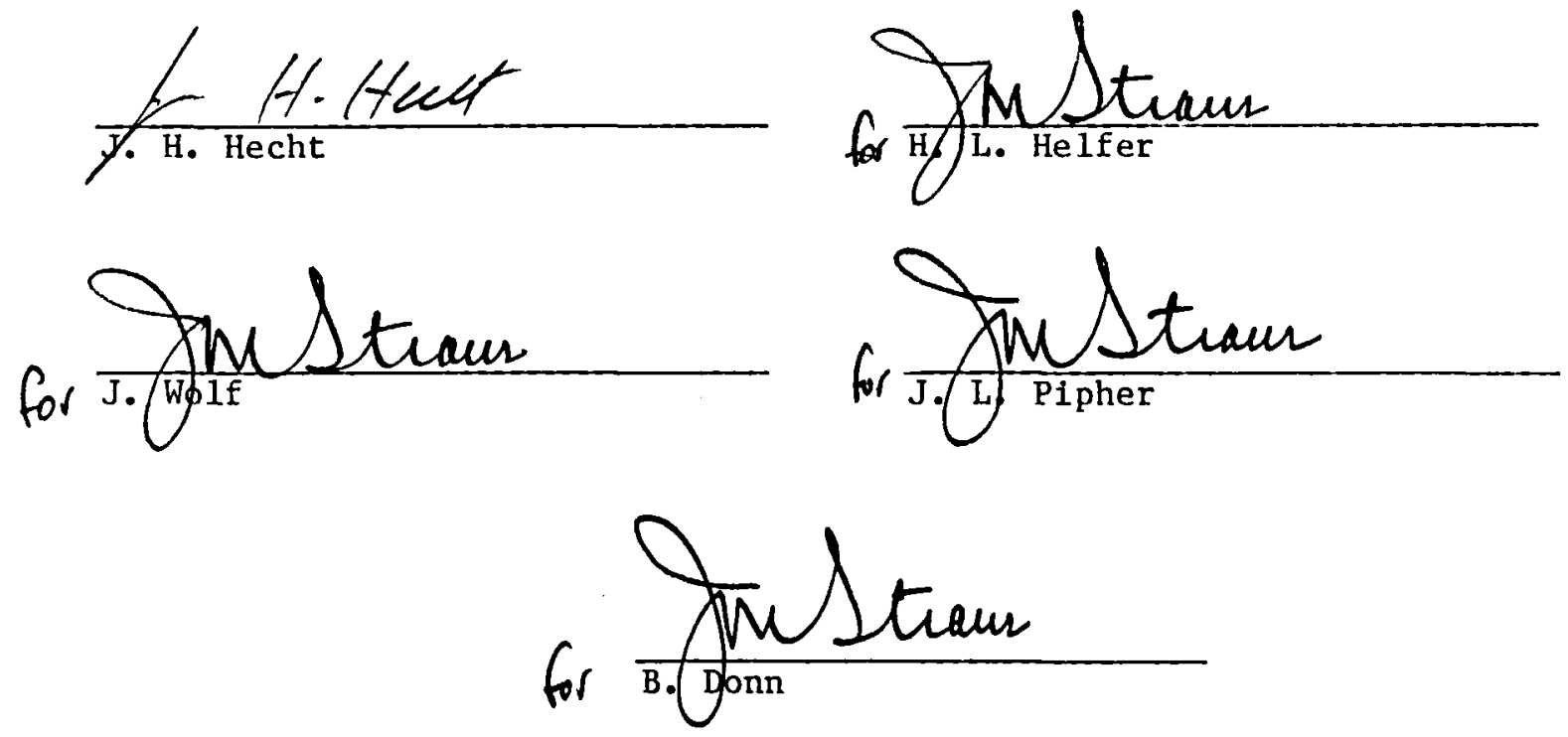

Approved
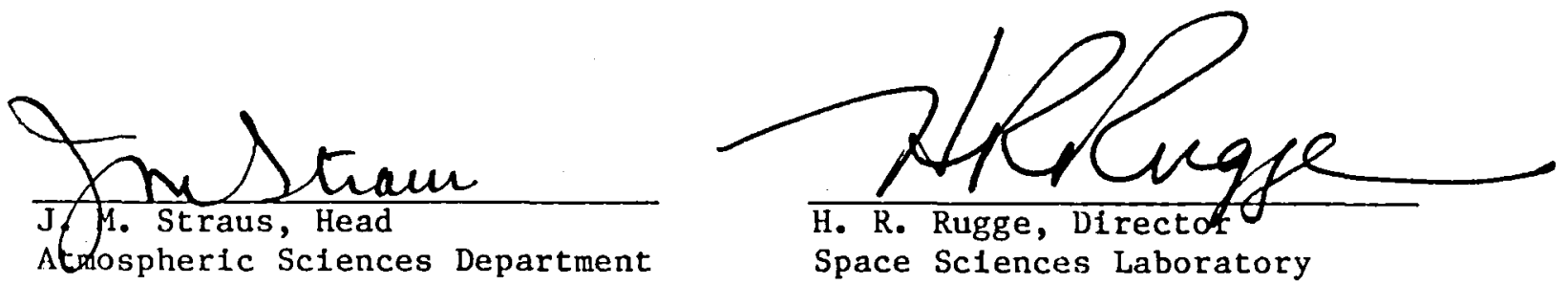
$-$

- 


\section{ABSTRACT}

The extinction of Herschel 36 has been measured and been found to be peculiar in the same sense as that observed in Orion. Following the treatment of Mathis and Wallenhorst, this can be explained by the presence of larger silicate and graphite grains than are normally found in the interstellar medium. Correcting the stellar flux for foreground extinction results in a residual extlaction curve for the assoclated dust cloud, with an unusually small normalized extinction (less than 1.0) at 1500 A. This low UV extinction may be due to the effects of scattering by the dust cloud material. 
ACKNOWLEDGMENTS

This work was supported 1n part by grant NAG5-46. Part of this data was obtained from the IUE Guest Investigator Program and part from the Astronomical Data Center at NASA/Goddard Space Flight Center. We wish to thank all supporting personnel at Goddard for their help and cooperation. The $U$ of $R$ and Goddard personnel wish to thank Dr. Al Boggess III for providing us with the SWP spectrum of Her 36 and for bringing together the Rochester and Goddard groups who were independently analyzing the same data. The results of both groups were combined for this paper. 
CONTENTS

ABSTRACT $\ldots \ldots \ldots \ldots \ldots \ldots \ldots \ldots \ldots \ldots \ldots \ldots \ldots \ldots \ldots \ldots \ldots \ldots \ldots \ldots, \ldots \ldots \ldots$

ACKNOWLEDGMENTS................................... vi

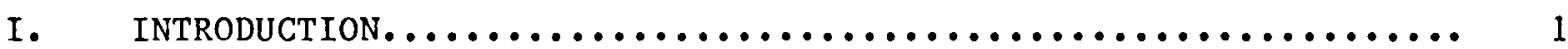

II. OBSERVATIONS AND DATA REDUCTION..................... 3

III. DISCUSSION $\ldots \ldots \ldots \ldots \ldots \ldots \ldots \ldots \ldots \ldots \ldots \ldots \ldots \ldots \ldots \ldots \ldots \ldots \ldots \ldots$

A. No Correction for Foreground Extinction.............. 7

B. Corrected for Foreground Extinction................ 9

IV. CONCLUSTONS $\ldots \ldots \ldots \ldots \ldots \ldots \ldots \ldots \ldots \ldots \ldots \ldots \ldots \ldots \ldots \ldots \ldots \ldots \ldots$

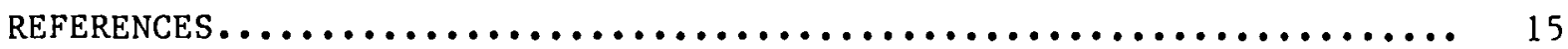

\section{FIGURES}

1. Extinction vs. Wavelength; the Shortest

Wavelength Shown is $1260 \AA$ \&......................... 4

2. Extinction vs. Wavelength Normalized

to the Peak at $2170 \AA$; the Shortest

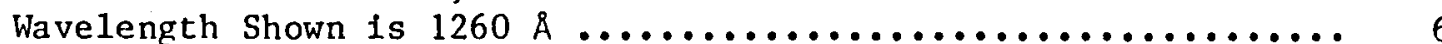


$-$

- 


\section{INTRODUCTION}

Thackeray (1950) and Woolf (1961) have suggested that the 0 star Herschel 36 (1950 coordinates, $\alpha=180036.2, \delta=-242252$ (Van Altena and Jones, 1972)), which might excite part of the M8 complex, may be embedded In dust. Johnson (1967) has shown that Her 36 has unusual UBVRIJKI photometry, similar to that found in the Orion Trapezium stars. Since the Orion stars are known to have pecullar extinction curves (Bohlin and Savage, 1981), we dectded to examine the UV extinction of Her 36.

The evidence for Her 36 being part of the M8 complex is strong. It is within $20^{\prime \prime}$ of both the Bourglass nebula and the $3-\mathrm{GHz}$ radio center of M8 (Turner et al., 1974) and there is a considerable [0 III] Ionfzation front structure near the star (Ellfot and Meaburn, 1975). An analysis underway Indicates that $\sim 90 \%$ of the 1300 - 1900 \& spectrum of the Hourglass can be attributed to reflection from Her 36 (Helfer et al., 1981). Recent nebular polarization studies by Lacasse et al. (1981) show evidence of large angle scattering in the vicinity of Her 36. Her 36 may be a member of NGC 6530 , the young $O B$ star cluster which appears associated with M8 (Van Altena and Jones, 1972); this cluster contalns $9 \mathrm{Sgr}$, an $04 \mathrm{~V}((f)$ ) star (Walborn, 1973) which is supposed to produce most of the fonization in M8 (Pottasch, 1965). UBV photometry of the cluster by Walker (1957) Indicates a distance of $1.4 \mathrm{kpc}$ and a fatrly unform foreground color excess of $E(B-V)=0.33$. Johnson found that Her 36 has an $E(B-V)$ of 0.88 vs. 0.33 for $9 \mathrm{SgI}$ and that Her 36 1s 4.36 visual mag fainter than $9 \mathrm{Sgr}$. The agreement of $E(B-V)$ values for NGC 6530, as a whole, and $9 \mathrm{Sgr}$ indicate that there is no shell surrounding that star. Circumstellar obscuration probably accounts for the faintness of Her 36 , since the intrinsic magnitude difference between main-sequence 0 stars is less than two magnitudes (Walborn, 1973). 


\section{OBSERVATIONS AND DATA REDUCTION}

We have examined low-resolution ( 6 A) spectra of Her 36 taken with the long (LWR 2485, taken 9/28/78) and the short (SWP 4222, taken 2/11/79) wavelength spectrographs of the IUE. The $10^{\prime \prime}$ by $20^{\prime \prime}$ IUE slit was oriented to exclude the Hourglass nebula. Using the linited number of early-type spectra published by Henize et al. (1975) for comparison, we find that the 1200 1700 \& spectrum is earller than 09 and of lower luminosity than a supergiant. (Si IV $1394 / 1403$ are strong but much weaker than CIV 1549, which, while strong, is not outstandingly so.) This is consistent with Woolf's (1961) 07 dwarf classification which we assume hereafter. There 1s no evidence that Her 36 is a vartable, although Walborn (1981) notes that $\theta^{1}$ or 1 C is a spectrum variable.

The differential extinction for Her 36 was calculated from

$$
\begin{aligned}
& 2.5 \log \left(F_{\lambda}(15 \text { Mon }) / F_{\lambda}(\text { Her } 36)\right)- \\
& (V(\text { Her 36) }-V(15 \text { Mon }))=E(\lambda-V)
\end{aligned}
$$

The data for the 07 comparison star 15 Mon $(\nabla=4.65, E(B-V)=0.07)$ was taken from two IUE spectra (LWR 7077, SWP 8146). These polats were corrected for reddening using the Savage and Mathls (1979) curve shown in F1g. 1a. The normalized extinction, $E(\lambda-V) / E(B-V)$, for Her 36 was calculated in two ways, from 1260 - $3000 \AA$, after foining the two spectra at $1950 \AA$. The first method, not correcting for any foreground extinction of Her 36, used the flux measurements described above, along with $E(B-V)$ equal to 0.88 and $V$ equal to 10.3 in equation 1. This result is shown in Fig. $1 \mathrm{~b}$. The second technique Involved dereddenting the flux of Her 36 corresponding to the assumed foreground differential absorption, $E(B-V)=0.33$, by using the Savage and Mathis 


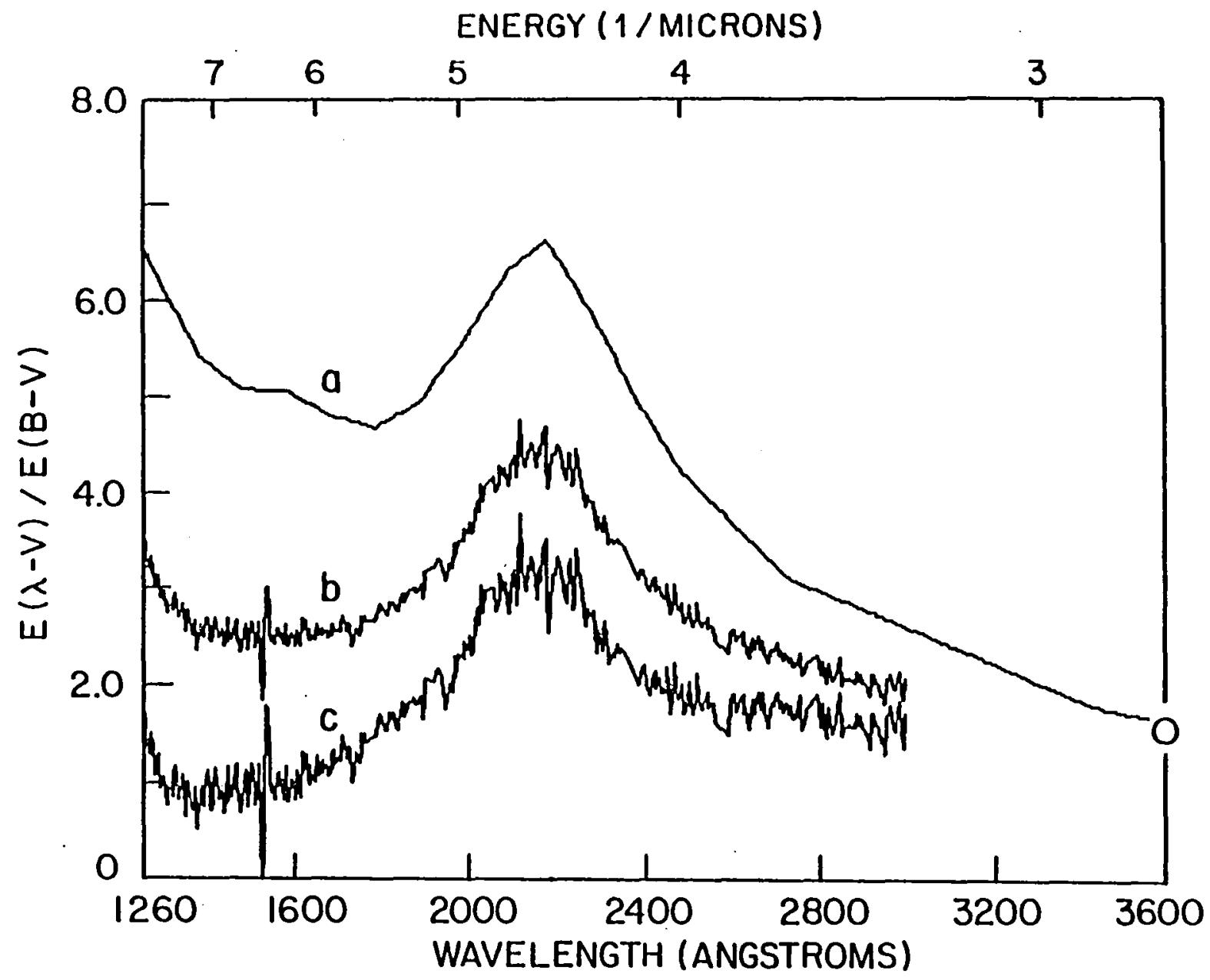

Fig. 1. Extinction vs. Wavelength; the Shortest Wavelength Shown is $1260 \AA$. (a) Normal interstellar extinction curve (Savage and Mathis, 1979). (b) Herschel 36 measured total extinction. (c) Herschel 36 with foreground extinction removed (see text). The open ctrcle value at $3600 \AA$ is from Johnson (1967) and is the same for (b) and for (c). 
curve (1979). Assuming a V of 9.3, corrected for 1 mag of foreground absorption, the residual extinction curve shown in Fig. Ic was calculated. The Rochester group found that these results are unchanged, within the noise, If $\lambda$ OrI (08V) or $10 \mathrm{Lac}(09 \mathrm{~V})$ were used as comparison stars. Both curves, 1b and 1c, can be extrapolated to Johnson's (1967) U photometry point, E(U$\mathrm{V}) / \mathrm{E}(\mathrm{B}-\mathrm{V})=1.66,1.64$, respectively. Following Mathis and Wallenhorst (1982) we also show, in F1g. $2, E(\lambda-V) / E(2170-V)$. 


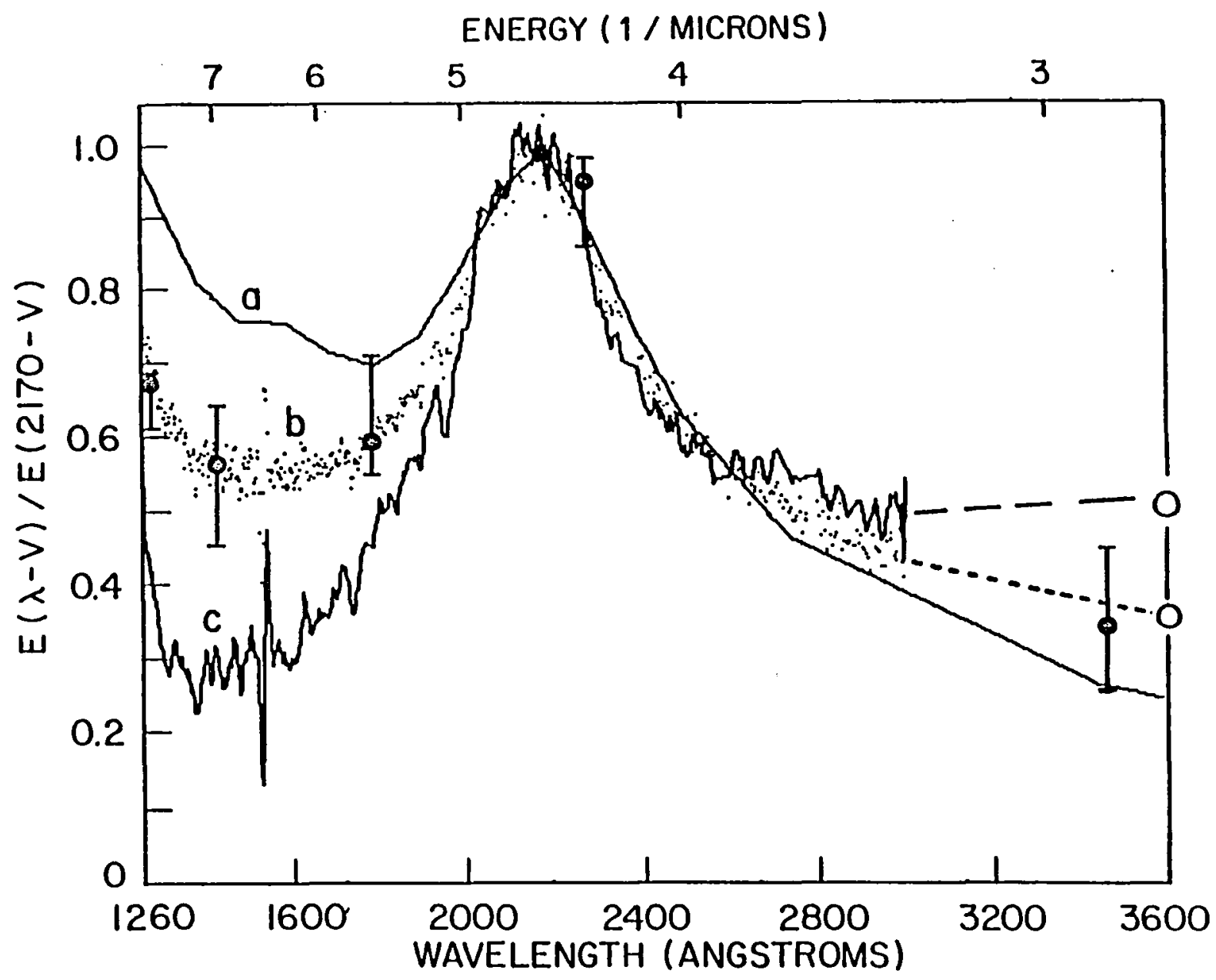

Fig. 2. Extinction vs. Wavelength Normalized to the Peak at $2170 \AA$; the Shortest Wavelength Shown is $1260 \AA$. (a)

Normal interstellar extinction curve (Savage and Mathis, 1979). (b) Herschel 36 measured total extinction (shown as dots). The open circle point at $3600 \AA$ is from Johnson (1967). The solid circles represent derived points from a mixture of silicate and graphite grains using the data from Mathis and Wallenhorst (1981). They apply only to curve b (see text). (c) Hersche1 36 with the foreground removed (see text). For clarity the data have been smoothed by averaging over $20-\AA$ intervals. The open circle point at $3600 \AA$ is from Johnson (1967). 


\section{DISCUSSION}

The existence of anomalous extinction curves is well established (see Meyer and Savage, 1981; Bohlin and Savage, 1981; Sitko, Savage and Meade, 1981; Witt, Bohlin and Stecher, 1981; Seab, Snow and Joseph, 1981; Greenstein, 1981; Snow and Seab, 1980; Wu, Gilra and Van Dulnen, 1980). However, only one quantitative model has been proposed to explain them (Mathis and Wallenhorst, 1981; hereafter $\mathrm{MW}$ ).

The Her 36 extinction curves are simflar to those in other dusty regions, e.g. $\rho$ Oph and Orion. As in these regions the extinction at the $2170 \AA$ bump Is weaker than normal (4.4 in Fig. Ib and 3.1 in FIg. 1c); the extinction shortward of 2170 A is much weaker, relative to normal extinction, than is the extinction near $3000 \AA$; and there is a far-UV rise only at wavelengths less than 1400 A, instead of the normal-UV rise near 1800 A. From Fig. Ib (Ic) we find that $E(2170-3300) / E(B-V)=2.7$ (1.4) compared to the normal value of 5.0 (Savage, 1975). These low values make Her 36 the most extreme member of the group $\theta$ Ori, NU Ori, $\sigma$ Sco and $\rho$ Oph. The position of the bump at 2170 \& appears normal.

\section{A. No Correction for Foreground Extinction (F1gs. 1b, 2b)}

It is of interest to see if the $M$ model is useful in interpreting our results. MW give the optical depth per $10^{22}$ \& atoms for different distributions of graphite and silfcate grains. We have fit curve b in Fig. 2 by simply adding their graphite and silfcate distributions, calculating $E(\lambda-V) / E(B-V)$, and normalizing the results to the $2170 \AA$ bump. (This assumes that carbon and silicon are equally depleted.) The best fit occurs when a 
$0.04-0.5 \mu \mathrm{m}$ silicate grain distribution is added to a $0.02-0.4 \mu \mathrm{m}$ graphite grain distribution. Also shown (as error bars) is the result of varying the graphite distribution from $0.01-0.4 \mu$ (bottom bar) to $0.04-0.5 \mu m$ (top bar). The normal interstellar extinction curve (Figs. 1a, 2a) is fit by using a $0.01-0.25 \mu \mathrm{m}$ distribution for both materials (Mathis, Rumpl and Nordsteck, 1977).

A value for $R$ (the ratio at 5500 \& of the total to selective extinction) can be calculated following Johnson (1967). The apparent magnitude difference between Her 36 and 9 Sgr is $4.36 \mathrm{mag}$. The absolute magnitude difference between an 07 star (Walborn 1973) and 9 Sgr (Humphreys, 1978) is 1.3 mag. We add the foreground extinction for $9 \mathrm{Sgr}, \mathrm{A}_{\mathrm{v}}=1.0$, to the difference and divide by the color excess for Her $36,0.88$, to get $R=4.6$. If the foreground extinction in front of $9 \mathrm{Sgr}$ also is in front of ther 36, accounting for 0.33 mag of the color excess, and we correct for it, we get $R=5.6$ for the residual extinction of the absorbing cloud around Her 36 . Even if Her 36 is subluminous by as much as I mag, as $\theta^{1}$ OrI $C$ may be (Walborn, 1981), both $R$ values are larger than the normal value, 3.1. MW state that a larger silicate or graphite maximum size is needed when $R$ is larger than 3.1 , and our best fit is in agreement with this statement.

MW found similar results for the silicate grain distribution in two other dusty regions near $\rho$ Oph and $\theta^{1}$ Orf $C$. The larger $R$ value correlates with the greater silfcate maximum size, while the absence of a Ilse in the normalized extinction at wavelengths less than $1800 \AA$ implies that the smaller silicates are absent. The rise at $1400 \AA$ is due to graphite and not to silicates, according to the optical depth table given in MW. The derived graphite grain distribution also has a larger average slze than normal, although the minimum size is less than $M$ found for $\theta^{1}$ orl $C$. However, since the bump position and 
width are normal, as in $\sigma$ Sco, the minimum size should have the usual interstellar value according to MW. This discrepancy may be related to the problem of the optical depth of the grains, which is discussed below.

\section{B. Corrected For Foreground Extinction (Fig. 1c, 2c)}

The normalized extinction, shown in Figs. $1 c$ and $2 c$, is similar to that found in the Orion region (Bohlin and Savage, 1981). Besides a weak (1c) and narrow $(2 c)$ bump $(E(2170-V) / E(B-V)=3.1)$, the most striking feature is that the minimum near $1500 \AA$ is slightly less than 1 , the value at $3000 \AA$. This can be compared to the MW curves by calculating a value of $X=E(2170-$ 3460)/E(2170-1430). The value of $X$ for the corrected Her 36 normalized extinction (Fig. 2c) is 0.7 . This value is accurate even if we consider how much our dereddening procedure would be affected by the regional variations in foreground extinction discussed by Meyer and Savage (1981). Following Massa et al. (1982) we can also use ANS data (Wesselius et al., 1982) at 1500, 2200, and $3300 \AA$ for another $M 8$ member star (HD164816 BO V, $E(B-V)=0.30)$, in order to estimate the variation from the Savage and Mathis curve. With this procedure our value for $X$ could be as high as 0.9 . The normal value of $X$ derived from the interstellar extinction curve (Fig. 2a) is 3.4. In order to understand this result, we note that the value of $X$ drops to 1.7 if the largest MW graphite and silicate distributions are used in computing the MW curves. Using only the largest graphite grain distribution, which does not peak at $2170 \mathrm{~A}$, reduces the value of $X$ to 1 , which is st1ll above the Her 36 result.

Part of the extinction may be due to the cloud of residual proto-stellar material surrounding Her 36 (Woolf, 1961). It is therefore necessary to take Into account not only photons absorbed or scattered by intervening dust grains, the effect of which is included in the MW curves, but also photons 
scattered into the fleld of view by nearby material. Also, if the materlal obscuring Her 36 is optically thick, multiple scattering effects must also be included. Since the cloud around Her 36 has an optical depth of 4.4 at 2170 A,derived from the $R$ value of 5.6 and the peak value of the excess in Fig. Ic, the multiple scattering effects are important. One possibility which would allow those effects to be estlmated is to assume that this pecullar extinction is caused by a circumstellar shell around Her 36. Mathis (1972) gave a formula, good to $15 \%$ accuracy for optical depths less than 4 , which allows the extinction by a dust shell to be calculated. We shall assume it is applicable, with lesser accuracy, for our range of $\tau$ in order to estimate the effects of multiple scatterling. To use the formula we first calculate from Mie theory the albedo, asymetry and optical depth (van de Hulst, 1957) for the varlous sizes of graphite and silicate grains included in the MW size distributions discussed previously. The dielectric constants for silicates were taken from Huffman and Stapp (1973), and those for graphite were taken from Taft and Philipp (1965) and Tosatt and Bassant (1970). Calculations using the Mathis formula Indicate that for an MW size distribution of silicate and graphite grains in a circumstellar shell, the value of $X$ will increase relative to the $M W$ result, $X \geq 1$, given above. Therefore, scatterlng by graphite and silicate grains in a circumstellar shell would not appear to be the sole cause of the extinction around Her 36 .

However, the dielectric constants of graphite are controversial (see Buffman, 1977; Hecht, 1981). If we use those values measured by Venghaus (1975), rather than those from Tosatti and Bassani (1970), we find, using the Mathis formula, that $X$ actually decreases to less than 1 for the larger graphite grain distributions. Because of the uncertainty in these calculations, it can only be stated that the dust could be predominantly $0.02-$ 
0.5 in graphite particles, with less than $5 \%$ of the optical depth artsing from $0.02-0.5 \mathrm{~m}$ silicate particles. It does not appear that the dust could have the normal interstellar mixture or even consist equally of silicates and graphite. The exact mixture cannot be uniquely determined, but clearly more graphite grains than sillcate grains are required. Calculations were also made using Code's (1973) slmple formula for radiative transfer through extended circumstellar material, and the results support these same general conclustons.

These calculations are only approximate since the exact effects of scattering will be affected by cloud geometry. However, since the optical depth of the cloud is large and most of the emergent photons have undergone multiple scattering, regardless of the detalls of cloud geometry, light scattering is important in understanding the extinction of Her 36. Thackeray (1950) has suggested that the dust associated with a wisp of bright nebulosity apparently connected to Her 36 might be the cause of its obscuration. Johnson (1967), however, found the nebula emission north and south of the star to be much fainter than the emission from the direction of the star. One possibility, consistent with our work and the above-mentioned studies, is that the peculiar extinction is due to light scattered from a shell of material concentrated around Her 36. 
- 
The graphite and silicate grains which are causing the extinction of Her 36 are larger than those found in the interstellar medium. This is consistent with the results of $\mathrm{MW}$. In particular, with the foreground extinction removed, the resultant cloud seems to be predominantly graphite, although some larger silicate grains may be present. The decrease in extinction at 1500 \& from $>2$ to a value of less than 1, after the removal of foreground extinction, may be caused by scattering effects from dust close to the star. This suggests that such effects may be important in the analysis of other cloud reglons. 
Bohlin, R. C. and Savage, B. D. 1981, Ap. J. 249, 109.

Code, A. D. 1973, IAU Symposium 52, Interstellar Dust and Related Topics, ed. J. M. Greenberg and H. C. van de Hulst (Dordrecht: Reldel) p. 505. Ell1ot, K. H. and Meaburn, J. 1975, MRRAS 172, 427. Greenstein, J. L. 1981, Ap. J. 245, 124 . Hecht, J. 1981, Ap. J. 246, 794.

Helfer, H. L., Pipher, J. L., Herter, T. and Wolf, J. 1981, private communicaElon.

Henize, K. G., Wray, J. D., Parsons, S. B. and Benedict, G. F. 1979, Catalog of Far UV Objective-Prism Spectroscopy (NASA Ref. Pub. No. 1031).

Huffman, D. R. 1977, Adv. Phys. 50, 129.

Huffman, D. R. and Stapp, J. L. 1973, IAU Symposium 52, Interstellar Dust and Related Topics, ed. J. M. Greenberg and H. C. van de Hulst (Dordrecht: Reide1) p. 297.

Humphreys, R. 1978, Ap. J. Supp1. $38,309$.

Johnson, H. L. 1967, Ap. J. 147, 912.

Lacasse, M. G., P1pher, J. I. and Sharpless, S. 1981, private communication. Massa, D., Savage, B. D. and F1tzpatrick, E. L., Ap. J., 1982 (submitted). Mathis, J. S. 1972; Ap. J. 176, 651. Mathls, J. S., Rumpl, W. and Nordsleck, K. H. 1977, Ap. J. 217, 425. Mathis, J. S. and Wallenhorst, S. G. 1981, Ap. J. 244, 483. Meyer, D. M. and Savage, B. D. 1981, Ap. J. 248, 545. Pottasch, S. R. 1965, Vistas in Astronomy 6. Savage, B. D. 1975, Ap. J. 199, 92. Savage, B. D. and Mathis, J. S. 1979, Ann. Rev. Astr. Ap. 17, 73. 
Seab, C. G., Snow, T. P. and Joseph, C. L. 1981, 246, 788.

Sitko, M. L., Savage, B. D. and Meade, M. R. 1981, Ap. J. 246, 161.

Snow, T. P. and Seab, C. G. 1980 , Ap. J. 242, L83.

Taft, E. A. and Philipp, H. R. 1965, Phys. Rev. 138, Al97.

Thackeray, A. D. 1950 , M. N. R. A. S. $110,343$.

Tosatt1, E. and Bassani, F. 1970, Nuovo Cimento 65B, 161.

Turner, B. E., Ballck, B., Cudaback, D. D., He1les, C. and Boyle, R. J. 1974, Ap. J. 194, 279.

Van Altena, W. F. and Jones, B. F. 1972, Astron, and Astroph. 20, 425.

van de Hulst, H. C. 1957, Light Scattering by Small Particles (London: Wiley).

Venghaus, H. 1975, Phys. Status. Solidi B 71, 609 .

Walborn, N. 1973, A. J. $78,1067$.

Walborn, N. 1981, Ap. J. (Letters) 243 , L37.

Wesselius, P. R., van Duinen, R. J., de Jonge, A. R. W., Aalders, J. W. G., Luinge, W. and W1ldeman, K. J., 1982, Astr. Ap. Suppl. (submitted).

Walker, M. F. 1957 , Ap. J. $125,636$.

Witt, A. N., Bohl1n, R. C. and Stecher, T. P. 1981, Ap. J. 244, 199.

Woolf, N. J. 1961, Pub. A. S. P. 73, 206.

Wu, C. C., Gilra, D. R. and van Duinen, R. J. 1980, Ap. J. $241,173$. 

(4) 